\title{
ULTRASONIC STUDIES OF POLYVINYLCHLORIDE IN CYCLOHEXANONE AND 1,4-DIOXANE
}

\author{
B. Thapa*, P. Shrestha** and P. Bhattarai*** \\ *Dept of Physics and Institute for functional Nanomaterials, University of Puerto Rico, San Juan, USA. \\ **Department of Chemistry, Padmakanya Campus, Bagbazar, Kathmandu, Nepal. \\ ***Department of Physics, Padmakanya Campus, Bagbazar, Kathmandu, Nepal.
}

\begin{abstract}
Ultrasonic velocity and density were measured for the binary systems: polyvinylchloride — cyclohexanone and polyvinylchloride-1,4-dioxane. And hence, the acoustic impedance $(Z)$ and reciprocal of adiabatic compressibility $\left(\beta^{-1}\right)$ were computed. The variations of ultrasonic velocity, density and acoustic impedance with concentration of polyvinylchloride were studied; confirming the absence of solute-solvent interaction.
\end{abstract}

Keywords: Condensed matter; Polymer-solvent; Ultrasonic velocity.

\section{INTRODUCTION}

Polyvinylchloride (PVC) is a well known versatile thermoplastic commodity, whose production and consumption is second worldwide compared to other plastics. PVC is generally known to have the advantages of low ingredient cost, wide processing versatility, high decorative potential and is used to manufacture various types of products ranging from highly rigid to very flexible [1]. The massive studies on polymers have led to several applications in polymer science. Many investigations were made for the study of ultrasonic velocity in polymer/solvent systems [2-7]. However, the investigation on polyvinylchloride is limited and it is interesting to pursue research on it. Thakore et al. [8] studied the compatibility of blend system of PVC and starch acetate in 1,4-dioxane from viscometric, ultrasonic and density measurements.

The viscosity of dilute polyvinylchloride solution was observed by Takeda and Endo [9]. Similarly, Xiang et al. [10] explored an effective means of characterizing structural changes of $\mathrm{PVC}$ particles during gelation and fusion of PVC plastisols with small-angle light scattering (SALS). Presently, we have carried out the investigation on PVC using two binary systems: polyvinylchloridecyclohexanone and polyvinylchloride - 1,4-dioxane to determine the density $(\rho)$ and ultrasonic velocity (v) at different concentration of PVC at constant temperature $25^{\circ} \mathrm{C}(298 \mathrm{~K})$.

\section{OBJECTIVES}

Objective of this study is to calculate different thermoacoustical parameters like reciprocal of adiabatic compressibility, acoustic impedance and to see the presence of solute solvent interaction.

\section{EXERIMENTAL SECTION}

Materials: The chemicals employed were purchased from Glaxo India Limited (Mumbai, India), manufactured by Qualigens Fine Chemicals (respective product no. 18265 and 18365 for cyclohexanone and 1,4-dioxane). The polymer used for study is polyvinylchloride resin manufactured by Thai Plastic and Chemicals Public Company ltd. (http://www.thaiplastic.co.th). The samples have been used without further purification. The characteristics of the corresponding materials are listed in Table 1. In the density measurements of binary system, the concentration of PVC was gradually increased.

Table 1: Characteristics of "cyclohexanone, 1,4-dioxane and ${ }^{\mathrm{P} p o l y v i n y l c h l o r i d e, ~ w h e r e ~} \mathrm{M}_{\mathrm{w}}$ and $\rho$ stands for molecular weight and density of the respective material in amu and $\mathrm{g} / \mathrm{cc}$ respectively as indicated.

\begin{tabular}{llllll}
\hline $\mathrm{M}_{\mathrm{w}}$ & $\mathrm{M}_{\mathrm{w}}$ & ${ }^{\mathrm{P}} \mathrm{M}_{\mathrm{w}}$ & ${ }^{\ddagger} \rho_{\mathrm{w}}$ & $\rho$ & ${ }^{\mathrm{P}} \rho$ \\
\hline 98.15 & 88.11 & 6720 & 0.946 & 1.030 & 1.44 \\
\hline
\end{tabular}

\section{METHODS}

The instrument used for this experiment is variable path interferometer (Mittel-84) with an accuracy of $0.3 \%$, manufactured by Mittel Enterprises, New Delhi, India. In order to prepare the binary system for polyvinylchloride in its solvents, a known amount of polyvinylchloride was weighed by using digital balance (Sartorious GMBH GOTTINGEN, Germany) and dissolved in a known amount of solvents: cyclohexanone and 1,4-dioxane. The density was measured with the help of specific gravity bottle of known volume. The basic principle involved in measuring the ultrasonic velocity

Author for Correspondence: Pradeep Bhattarai, Department of Physics, Padmakanya Campus, Bagbazar, Kathmandu, Nepal. E-mail:bhattaraipradeep@hotmail.com. 
with a single frequency $(1 \mathrm{MHz})$ in liquids is based on setting up of resonance by stationary waves in a column limited by the source at one end and a reflector at the other end in the interferometer.

At resonance, the anode current in the generator of interferometer rises to a maximum value. A number of such resonances can be observed by increasing or decreasing the distance between the plates in steps of one half wavelengths by means of the micrometer arrangement. If $d$ is the total distance moved and $n$ be the number of maxima, then the condition for the resonance (i.e., maximum anode current) is $d=n \lambda / 2$, where, ' $\lambda$ ' is the wavelength of ultrasonic wave. Now, the velocity of the ultrasonic wave of wavelength $\lambda$ in the liquid is given by $v=\lambda f$. So, $v=2 d f / n$. Knowing the value of ultrasonic speed (v) in a medium, the acoustic impedance $(Z)$ and the reciprocal of adiabatic compressibility $\left(\beta^{-1}\right)$ for the medium can be calculated with the aid of following relations $Z=\rho v$ and $\beta^{-1}=\rho v^{2}$ where, $\rho$ being the density of the solution.

\section{RESULTS AND DISCUSSION}

Density ( $\rho)$, Ultrasonic velocity (v), acoustic impedance (Z) and reciprocal of adiabatic compressibility $\left(\beta^{-1}\right)$ at $298 \mathrm{~K}$ for binary systems. Density $(\rho)$, ultrasonic velocity (v) for various concentrations of PVC in cyclohexanone and 1,4-dioxane at $25^{\circ} \mathrm{C}(298 \mathrm{~K})$ are measured. Physical quantities like acoustic impedance $(Z)$ and reciprocal of adiabatic compressibility $\left(\beta^{-1}\right)$ are calculated using the equations mentioned above. From Figure 1, we determined two distinct values for the Ultrasonic velocity in polyvinyl chloride: 2.49635 and $2.18715 \mathrm{~km}$ $\mathrm{sec}^{-1}$ respectively contributed by polyvinylchloridecyclohexanone and polyvinylchloride-1,4-dioxane systems. This slight deviation in the value of ultrasonic velocity is due to the contributions of different nature of the components in the solutions (i.e. the former one is the contribution of polar-polar system of components while the later is the contribution of polar-non-polar system of components). Table 2 shows experimentally determined ultrasonic velocity and density, calculated values of acoustic impedance and reciprocal of adiabatic compressibility of polyvinylchloride cyclohexanone and polyvinylchloride - 1,4-dioxane binary system with different concentration of polyvinylchloride.

Velocity increases in the system with the increase of concentration of PVC. Gurung et al. [3] have concluded the similar result for the binary system of polystyrene/ toluene. The linear dependency of the ultrasonic velocity on concentration was also observed by Helne and Snyder [11] and Regmi et al. [4]. Likewise velocity, the acoustic impedance in the system also increases with the concentration of PVC. This is the same result as presented by Rao et al. [7] for polyvinyl pyrrolidone and N,N-dimethylformamide solution. Rajulu et al. [12] have reported structural changes in the solution of polyethyleneglycol-300 and water on the basis of ultrasonic studies. But this investigation concludes no structural changes in the experimental systems caused by the solvents over the range of concentration of PVC studied. In figure 2 it is seen that numerically at 0.146814 concentration of polyvinylchloride, both the systems acquire the same velocity $1.483975 \mathrm{kms}^{-1}$. This peculiar behavior of polyvinylchloride in the binary systems is the most interesting part of this investigation.

Table 2: Experimentally measured density and ultrasonic velocity together with thermoacoustic parameters $Z$ and 及-1 with different concentration of Polyvinylchloride -Cyclohexanone.

\begin{tabular}{|c|c|c|c|c|}
\hline $\mathrm{f}_{1}(\mathrm{~g} / \mathrm{l})$ & $\rho(g / c c)$ & $\mathrm{V}(\mathrm{km} / \mathrm{sec})$ & $\mathrm{Z}(\mathrm{g} \mathrm{km} / \mathrm{cc} \mathrm{s})$ & $\beta^{-1}\left(\mathrm{~g} \mathrm{~km}^{2} / \mathrm{cc} \mathrm{s}^{2}\right)$ \\
\hline 0.00000 & $0.9471 \pm 0.01$ & $1.3113 \pm 0.0036$ & $1.2419 \pm 0.012$ & $1.62855 \pm 0.02$ \\
\hline 0.00069 & $0.9490 \pm 0.01$ & $1.3116 \pm 0.0036$ & $1.2447 \pm 0.012$ & $1.63256 \pm 0.02$ \\
\hline 0.00138 & $0.9523 \pm 0.01$ & $1.3124 \pm 0.0036$ & $1.2498 \pm 0.012$ & $1.64024 \pm 0.02$ \\
\hline 0.00208 & $0.9552 \pm 0.01$ & $1.3133 \pm 0.0036$ & $1.2545 \pm 0.012$ & $1.64749 \pm 0.02$ \\
\hline 0.00277 & $0.9588 \pm 0.01$ & $1.3143 \pm 0.0036$ & $1.2602 \pm 0.013$ & $1.65622 \pm 0.02$ \\
\hline 0.00346 & $0.9621 \pm 0.01$ & $1.3152 \pm 0.0036$ & $1.2654 \pm 0.013$ & $1.66419 \pm 0.02$ \\
\hline 0.00414 & $0.9660 \pm 0.01$ & $1.3161 \pm 0.0036$ & $1.2714 \pm 0.013$ & $1.67323 \pm 0.02$ \\
\hline 0.00483 & $0.9693 \pm 0.01$ & $1.3169 \pm 0.0036$ & $1.2765 \pm 0.013$ & $1.68098 \pm 0.02$ \\
\hline 0.00552 & $0.9719 \pm 0.01$ & $1.3178 \pm 0.0036$ & $1.2808 \pm 0.014$ & $1.68780 \pm 0.02$ \\
\hline 0.00621 & $0.9741 \pm 0.01$ & $1.3187 \pm 0.0037$ & $1.2845 \pm 0.014$ & $1.69393 \pm 0.02$ \\
\hline 0.00689 & $0.9781 \pm 0.01$ & $1.3201 \pm 0.0037$ & $1.2912 \pm 0.014$ & $1.70450 \pm 0.02$ \\
\hline 0.01369 & $1.0055 \pm 0.01$ & $1.3265 \pm 0.0037$ & $1.3338 \pm 0.014$ & $1.76928 \pm 0.02$ \\
\hline 0.02041 & $1.0255 \pm 0.01$ & $1.3325 \pm 0.0038$ & $1.3665 \pm 0.014$ & $1.82083 \pm 0.02$ \\
\hline 0.02702 & $1.0561 \pm 0.01$ & $1.3431 \pm 0.0038$ & $1.4184 \pm 0.014$ & $1.90512 \pm 0.02$ \\
\hline \multicolumn{5}{|c|}{ polyvinylchloride $-1,4$-dioxane } \\
\hline 0.00000 & $1.0330 \pm 0.01$ & $1.3620 \pm 0.0036$ & $1.4069 \pm 0.014$ & $1.91626 \pm 0.02$ \\
\hline 0.00069 & $1.0333 \pm 0.01$ & $1.3627 \pm 0.0036$ & $1.4080 \pm 0.014$ & $1.91873 \pm 0.02$ \\
\hline 0.00138 & $1.0335 \pm 0.01$ & $1.3635 \pm 0.0036$ & $1.4092 \pm 0.014$ & $1.92147 \pm 0.02$ \\
\hline 0.00208 & $1.0338 \pm 0.01$ & $1.3644 \pm 0.0036$ & $1.4105 \pm 0.014$ & $1.92451 \pm 0.02$ \\
\hline 0.00277 & $1.0341 \pm 0.01$ & $1.3652 \pm 0.0036$ & $1.4117 \pm 0.014$ & $1.92727 \pm 0.02$ \\
\hline 0.00346 & $1.0343 \pm 0.01$ & $1.3661 \pm 0.0036$ & $1.4129 \pm 0.014$ & $1.93029 \pm 0.02$ \\
\hline 0.00414 & $1.0346 \pm 0.01$ & $1.3668 \pm 0.0036$ & $1.4141 \pm 0.014$ & $1.93278 \pm 0.02$ \\
\hline 0.00483 & $1.0349 \pm 0.01$ & $1.3677 \pm 0.0036$ & $1.4154 \pm 0.014$ & $1.93583 \pm 0.02$ \\
\hline 0.00552 & $1.0351 \pm 0.01$ & $1.3686 \pm 0.0037$ & $1.4167 \pm 0.014$ & $1.93888 \pm 0.02$ \\
\hline 0.00621 & $1.0354 \pm 0.01$ & $1.3694 \pm 0.0037$ & $1.4178 \pm 0.014$ & $1.94164 \pm 0.02$ \\
\hline 0.00689 & $1.0357 \pm 0.01$ & $1.3702 \pm 0.0037$ & $1.4191 \pm 0.014$ & $1.94439 \pm 0.02$ \\
\hline 0.01369 & $1.0383 \pm 0.01$ & $1.3718 \pm 0.0037$ & $1.4243 \pm 0.014$ & $1.95389 \pm 0.02$ \\
\hline 0.02041 & $1.0409 \pm 0.01$ & $1.3782 \pm 0.0038$ & $1.4345 \pm 0.014$ & $1.97710 \pm 0.02$ \\
\hline 0.02702 & $1.0433 \pm 0.01$ & $1.3866 \pm 0.0038$ & $1.4469 \pm 0.014$ & $2.00622 \pm 0.02$ \\
\hline
\end{tabular}




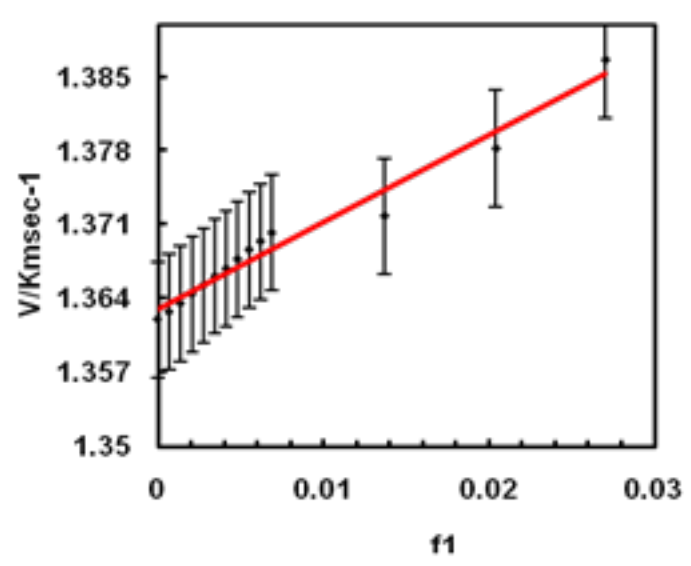

polyvinylchloride-cyclohexanone

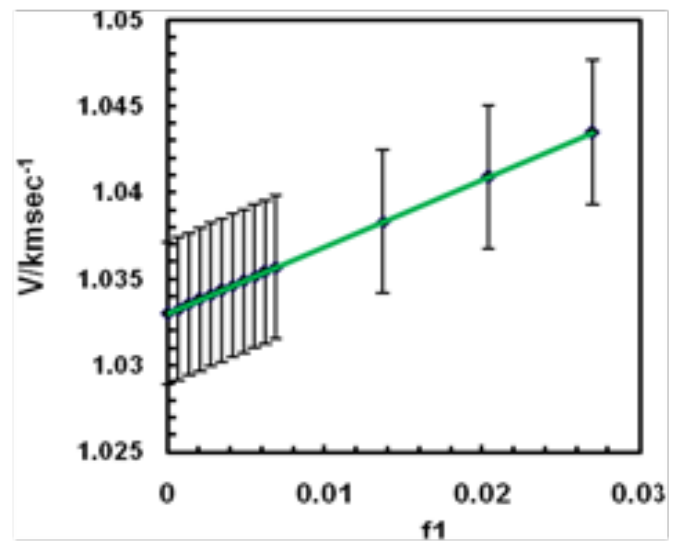

polyvinylchloride-1,4-dioxane

Figure 1: Concentration dependencies of ultrasonic velocity for binary systems.

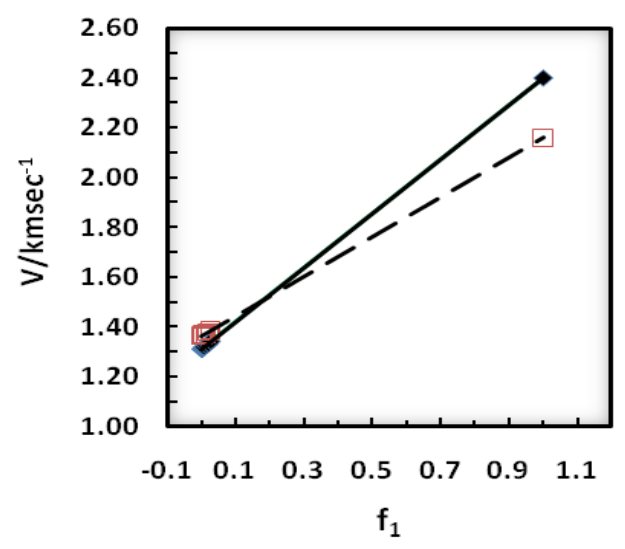

[( $)$ polyvinylchloride-cyclohexanone and ( $\square$ ) polyvinylchloride-1,4-dioxane ]

Figure 2: Variation of ultrasonic velocity with concentration of polyvinyl chloride for binary systems.

\section{CONCLUSIONS}

Tabhane et al.[13] study the missibility and interaction in polyvinyl chloride-polyvinyl acetate blend using Automatic Ultrasonic Attenuation Recorder (AVAR 102, and frequency counter APLAB-1116 and studied the nature of solvent polymer-polymer interaction and concluded that the nonlinear variation of ultrasonic velocity and other acoustical parameters suggest heterogeneity indicated by double-phase separation in the blend. In present experiment it has been found that the linear variation of ultrasonic velocity in the binary systems of polyvinylchloride-cyclohexanone and polyvinylchloride-1,4-dioxane with the concentration of polyvinylchloride. This study reveals the absence of solute-solvent interaction in the binary systems. It will be more fascinating to implement further investigations on polymer-polymer blend system in one or multi solvent system.

\section{ACKNOWLEDGEMENT}

Appreciation is expressed to Professor D. R. Mishra for his constructive suggestions and department head of Central Department of Physics, Professor L. N. Jha, for his profound support. P.B and P.S wish to express our gratitude to University Grants Commission, Nepal and Padmakanya Multiple Campus for their financial support to participate in the XVII NSFD 2012 in Bhubaneshwor, India. Thanks to IOST Dean's office Tribhuvan University for providing leave to participate in the conference.

\section{REFERENCES}

[1] Plastisols; Encylopedia of Chemical Technology,; 1983. 3rd ed., Wiley, New York. 23: 922 .

[2] Prasai, B. K.; Bhattarai, P. and Mishra, D. R. 2000. in Different Disordered-Systems. INDIAS Publications: Allahabad, India. p. 78-81.

[3] Gurung, T., Shakya, P. and Bhattarai, P. 1998. in Condensed Matter Physics, B. K. Agrawal and H. Prakash ((Eds), Narosa Publishing House, Delhi, India. p. 297-300.

[4] Regmi, S., Bhattarai, P. and Byahut, S. 2006. J. Polym. Matter. 23: 171-175.

[5] Rajulu, A., Varada and Mabu Sab, P., 1996. J.Eur. Polym. 32 (2): 267-268.

[6] Rajulu, A., Varada., Sreenivasulu, G. and Raghuraman, K. S. 1994. Pure Appl. Ultrason. 16: 29-33.

[7] Chowdoji Rao, K., Naidu, S. Venketa and Rajulu, A. Varada. 1990. Eur. Polym. J. 26 (6): 657-659.

[8] Thakore,I. M., Desai, Sonal., Sarwade, B. D. and Surekh Devi. 1999. Journal of Applied Polymer Science. 71 (11): 1851-1861.

[9] Takeda, Masatami and Endo, Ryuichi. 1956. Viscosity of dilute polyvinylchloride solution, Vol. 60, Tokyo College of science, Tokyo, Japan.

[10] Xian, Guang-ming., Ping, Jin. and Zeng, Bi-qing. 2008. Journal of Automated Methods and Management in Chemistry, Volume (2008), Article ID 838412, p.10.

[11] Helne J. C. and Snyder W. J. 1984. Am. Chem. Soc. 175.

[12] Rajulu, A.Varada, Sab, P. Mabu., Reddy, R. Lankshminarayana., Gurung, S. and Bhattarai, P. J. 1997. of Nep. Chem. Soc. 16, p.1.

[13] Tabhane, P. V., Chimankar O. P., Shriwas R. S., Dudhe, C. M. and Tabhane, V. A. 2012. Material science and engineering 42, 012033, IOP conference series, IOP publishing ltd. 